\title{
A novel approach to image reconstruction and calibration for a multi-slit-slat SPECT system
}

\author{
Kjell Erlandsson, Debora Salvado, Brian F Hutton on behalf of the INSERT collaboration
}

\begin{abstract}
In the context of the development of a simultaneous SPECT/MRI system, we have previously proposed a multi-minislit-slat (MSS) collimator, with multiple sections of short slits in order to improve the angular sampling. The data can be reconstructed using a 3D reconstruction algorithm that models the collimator geometry. One drawback, however, is that the projection data obtained with this collimator are difficult to interpret visually. Also, calibration can be problematic, as each mini-slit only covers part of the object FoV. We have therefore developed an algorithm for transforming the MSS projection data into the traditional sinogram format. These sinograms consist of multiple thin tilted lines with gaps in between due to the lack of detector rotation in this system. The data can be reconstructed using standard parallel-beam algorithms, taking into account the fact that there are data missing. We have shown with simulations and measurements that the algorithm can transform complex data, consisting of multiple rough broken line segments, into simple sine-curves. This algorithm can be useful for interpreting the acquired MSS data, reconstructing images, and calibrating the system.
\end{abstract}

\section{INTRODUCTION}

$\mathrm{T}$ HE aim of the INSERT project [1] has been the development of a SPECT insert for commercially available MRI scanners, primarily for the study of brain tumours. The fundamental unit of the INSERT system is a compact gamma camera with high intrinsic resolution [2], allowing for the use of diverging collimators with minification. One drawback of this system is the limited angular sampling, as no rotation is used for practical reasons. After investigating various collimator options [3], we decided to use the multi-mini-slitslat (MSS) collimator [4] (Fig. 1).

The MSS collimator consists of multiple, radially offset sections of short slits in order to improve the angular sampling (Fig. 2). The acquired data can be reconstructed using an algorithm we developed, based on angular blurring [5]. However, the projection data can be difficult to interpret visually, and calibration can also be problematic, as each minislit only covers part of the field-of-view (FoV). One way to address these issues would be to rebin the data into standard parallel projection format. However, the problem with this approach is that the angular sampling between detectors is relatively poor compared to that within each mini-slit

Manuscript received Nov 30, 2016. This work was funded by the EC under the FP7-HEALTH Programme (Grant number 305311) and supported by the National Institute for Health Research University College London Hospitals Biomedical Research Centre. D. Salvado is supported by a PhD Fellowship (FCT:SFRH/BD/88093/2012)

All authors are with The Institute of Nuclear Medicine, University College London, London, UK. B. F. Hutton is also with The Centre for Medical Radiation Physics, University of Wollongong, Australia. Corresponding author: K. Erlandsson (e-mail: k.erlandsson@ucl.ac.uk). projection. We have nevertheless implemented a rebinning algorithm, in which we simply ignore this problem, and assume a high angular sampling, which results in sinograms with a large number of gaps.

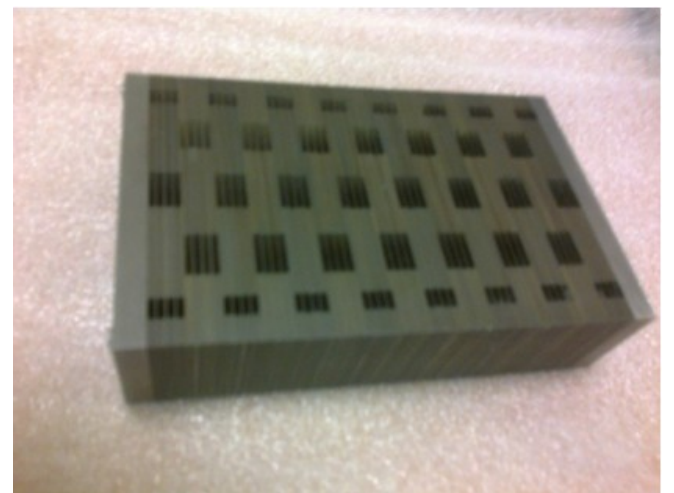

Fig. 1. Photograph of a prototype MSS collimator, manufactured by Nuclear Fields (NL).

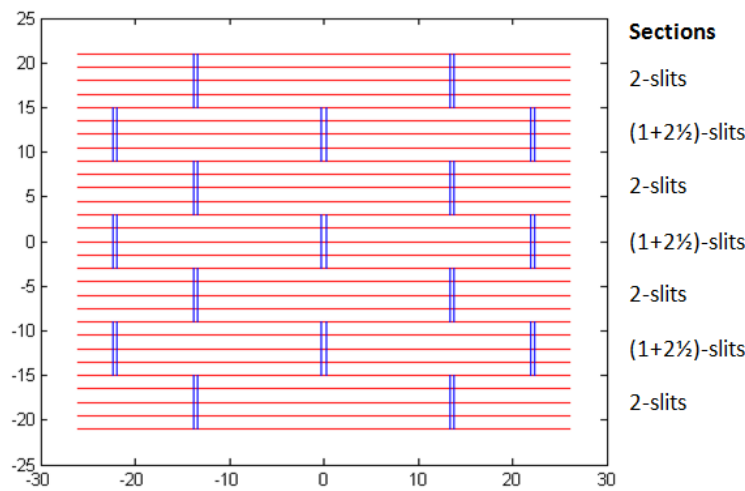

Fig. 2. Cross-section of MSS collimator, where the red horizontal lines represent the slats and the blue vertical lines the slits. This collimator has four 2 -slit sections and three $\left(1+2^{1 / 2}\right)$-slit sections (see [4] for details).

\section{METHODS}

\section{A. Reconstruction and Calibration}

Image formation in SPECT can be described by the following expression:

$$
\boldsymbol{g}=\mathcal{H} \boldsymbol{f}+\boldsymbol{n}
$$

where $\boldsymbol{g}$ is the acquired data, $\mathcal{H}$ is the system matrix, $\boldsymbol{f}$ is the activity distribution in the object, and $\boldsymbol{n}$ is a random noise term. Reconstruction is the process of inverting (1) to find the most likely $\boldsymbol{f}$ for a certain $\boldsymbol{g}$ when $\mathcal{H}$ is known. Calibration is the process of estimating $\mathcal{H}$ for a particular scanner as accurately as possible, assuming $\boldsymbol{f}$ and $\boldsymbol{g}$ are known, in order to minimise artifacts and maximise image quality. Our new rebinning method can be useful for both reconstruction and calibration. 


\section{B. Rebinning}

Sinograms were generated, using the following formulae:

$$
\left\{\begin{array}{l}
r^{\prime}=(R-F) \cdot \sin \left(\phi_{1}\right)-r_{S} \cdot \cos \left(\phi_{1}\right) \\
\phi^{\prime}=\phi_{0}+\phi_{1}
\end{array}\right.
$$

where $r^{\prime}$ and $\phi^{\prime}$ are the sinogram coordinates for a line-ofresponse (LOR) defined by the camera angle $\phi_{0}$ and the detector angle $\phi_{1}$, and $R$ is the detector radius, $F$ the focal length and $r_{S}$ the lateral slit-position (Fig. 3).

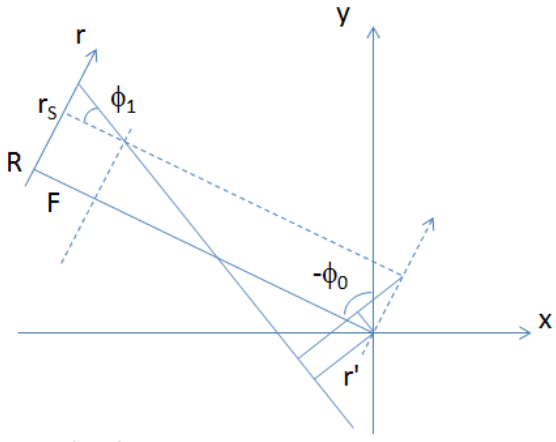

Fig. 3. Collimator \& LOR geometry.

\section{Evaluation}

We simulated data corresponding to an INSERT detector module equipped with a MSS collimator for 30 angles over $360^{\circ}$ with a source distribution consisting of 4 line sources placed at radial distances of 25, 50,75 and $100 \mathrm{~mm}$ from the centre-of-rotation (CoR) at $90^{\circ}$ intervals.

We processed the data to generate sinograms as indicated above with an angular sampling of $1^{\circ}$, using the correct geometric parameters, and also incorrect parameters. Images were reconstructed from the sinograms with OS-EM [6]. Data were also acquired with a prototype detector and a prototype MSS collimator. Sinograms were generated with optimised and non-optimised parameters.

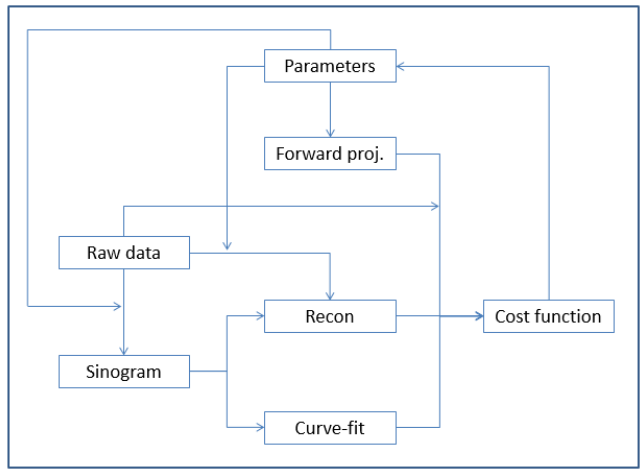

Fig. 4. Illustration of various calibration approaches.

\section{Calibration}

Geometric calibration of multi-pinhole SPECT systems is usually done by forward-projection of known source positions, based on assumed parameters, and comparison with measured data [7].

An alternative might be to reconstruct the raw data, based on assumed parameters, and compare with the known source positions. Using the sinogram rebinning technique, two new calibration approaches are possible. In both cases, the first step is to use the assumed parameters for generating the sinograms from the raw data. The second step can be to either reconstruct the sinogram-data or to simply fit these with sin-curves [8]. The various calibration approaches are illustrated in Fig. 4.

\section{RESULTS \& DISCUSSION}

\section{A. Rebinning}

Simulated projection data are shown in Fig. 5, displayed as pseudo-sinograms (raw data), as well as rebinned sinograms. It can be seen that the two collimator sections produce quite different raw data patterns. On the other hand, the corresponding sinograms obviously represent the same curves, although the sampling is not the same. An enlarged view of the area marked by a dashed rectangle in the leftmost sinogram is shown in Fig. 6. It can be seen that each sinecurve consists of multiple small line segments, which are tilted at a certain angle. Each of the line segments correspond to the projection of a source through one slit aperture. The length of the line depends on the resolution of the system, determined by the width of the slit-aperture and the intrinsic detector resolution. In the middle sinogram in Fig. 5, the lines are closer together, showing that improved sampling is obtained by combination of data from the different collimator sections.
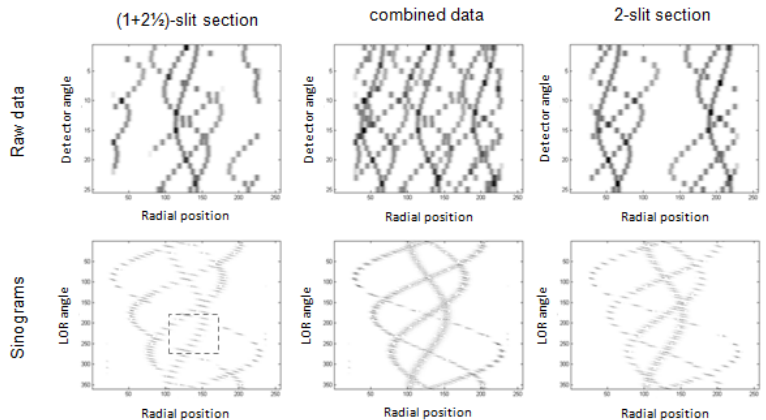

Fig. 5. Simulated data for 4 line-sources for two different collimator sections, including raw data and corresponding rebinned sinograms.

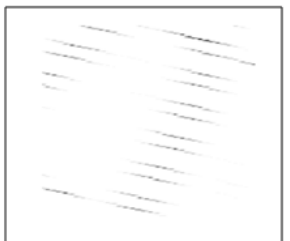

Fig. 6. Enlarged view of the area within the dashed rectangle in Fig. 5.

\section{B. Calibration}

Figure 7 shows an example of how rebinned sinograms can be used for calibration. It can be seen that the sinograms obtained with incorrect parameters are much broader than those obtained with correct parameters. This is obvious in both the simulated and real data. Also, the reconstructed linesources are mis-positioned and blurry when incorrect parameters are used. 


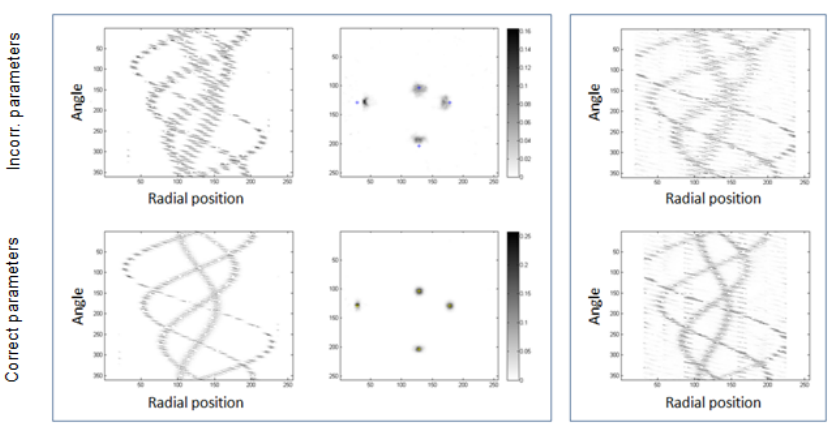

Fig. 7. Rebinned sinograms and reconstructed images obtained with incorrect and correct geometric parameters from simulated data (left panel) and real measured data (right panel).

\section{CONCLUSIONS}

We have proposed an algorithm for generating sinograms from data obtained with a novel multi-mini-slit-slat collimator. The resulting sinograms consist of multiple small tilted line segments due to the combination of coarse and fine angular sampling, between and within detectors, respectively. The rebinned sinograms can be useful for reconstruction and/or calibration.

\section{REFERENCES}

[1] P. Busca, C. Fiorini, A. D. Butt, M. Occhipinti, R. Peloso, R. Quaglia, et al., "Simulation of the expected performance of INSERT: A new multimodality SPECT/MRI system for preclinical and clinical imaging," Nuclear Instruments and Methods in Physics Research Section A: Accelerators, Spectrometers, Detectors and Associated Equipment, vol. 734, Part B, pp. 141-146, 1/11/ 2014.

[2] P. Busca, M. Occhipinti, P. Trigilio, G. Cozzi, C. Fiorini, C. Piemonte, et al., "Experimental Evaluation of a SiPM-Based Scintillation Detector for MR-Compatible SPECT Systems," IEEE Transactions on Nuclear Science, vol. 62, pp. 2122-2128, 2015.

[3] K. Erlandsson, D. Salvado, A. Bousse, and B. F. Hutton, "Design optimization and evaluation of a human brain SPECT-MRI insert based on high-resolution detectors and slit-slat collimators," in 2013 IEEE Nuclear Science Symposium and Medical Imaging Conference (2013 NSS/MIC), 2013, pp. 1-4.

[4] D. Salvado, K. Erlandsson, A. Bousse, M. Occhipinti, P. Busca, C. Fiorini, et al., "Collimator Design for a Brain SPECT/MRI Insert," IEEE Transactions on Nuclear Science, vol. 62, pp. 1716-1724, 2015.

[5] A. Bousse, K. Erlandsson, N. Fuin, D. Salvado, and B. F. Hutton, "Variance prediction in SPECT reconstruction based on the Fisher information using a novel angular blurring algorithm for computation of the system matrix," in 2013 IEEE Nuclear Science Symposium and Medical Imaging Conference (2013 NSS/MIC), 2013, pp. 1-6.

[6] H. M. Hudson and R. S. Larkin, "Accelerated image reconstruction using ordered subsets of projection data," IEEE Transactions on Medical Imaging, vol. 13, pp. 601-609, 1994.

[7] D. Beque, J. Nuyts, G. Bormans, P. Suetens, and P. Dupont, "Characterization of pinhole SPECT acquisition geometry," IEEE Transactions on Medical Imaging, vol. 22, pp. 599-612, 2003

[8] D. Salvado, K. Erlandsson, B. F. Hutton, "Effects of the Collimator Magnification Factor in the Geometrical Calibration of SPECT Systems", in 2016 IEEE Nuclear Science Symposium and Medical Imaging Conference (2016 NSS/MIC), 2016, pp. 1-4. 